\title{
BEPICOLOMBO - A MISSION TO MERCURY
}

\author{
R. Jehn*, J. Schoenmaekers, D. García and P. Ferri \\ European Space Operations Centre, ESA/ESOC, Darmstadt, Germany
}

\begin{abstract}
BepiColombo is a cornerstone mission of the ESA Science Programme, to be launched towards Mercury in July 2014. After a journey of nearly 6 years two probes, the Magnetospheric Orbiter (JAXA) and the Planetary Orbiter (ESA) will be separated and injected into their target orbits. The interplanetary trajectory includes flybys at the Earth, Venus (twice) and Mercury (four times), as well as several thrust arcs provided by the solar electric propulsion module. At the end of the transfer a gravitational capture at the weak stability boundary is performed exploiting the Sun gravity. In case of a failure of the orbit insertion burn, the spacecraft will stay for a few revolutions in the weakly captured orbit. The arrival conditions are chosen such that backup orbit insertion manoeuvres can be performed one, four or five orbits later with trajectory correction manoeuvres of less than $15 \mathrm{~m} / \mathrm{s}$ to compensate the Sun perturbations. Only in case that no manoeuvre can be performed within 64 days ( 5 orbits) after the nominal orbit insertion the spacecraft will leave Mercury and the mission will be lost.
\end{abstract}

The baseline trajectory has been designed taking into account all operational constraints: 90-day commissioning phase without any thrust; 30-day coast arcs before each flyby (to allow for precise navigation); 7-day coast arcs after each flyby; 60-day coast arc before orbit insertion; Solar aspect angle constraints and minimum flyby altitudes $(300 \mathrm{~km}$ at Earth and Venus, $200 \mathrm{~km}$ at Mercury). For the thrust arcs a duty cycle of $90 \%$ is assumed to provide some margin for thrust outages (e.g. due to safe modes) and for navigation manoeuvres. In the initial Earth-to-Earth arc, which is mainly needed to achieve the optimum departure declination, two thrust arcs are scheduled to increase the Earth flyby velocity with respect to the departure velocity. A trade-off is made between an initially outward or inward trajectory. From a delta-V point of view the outward option is slightly cheaper especially if a 30-day launch window is required. Also a backup trajectory with a launch in August 2015 will be shown in case the nominal launch will be delayed.

\footnotetext{
* Corresponding author

Email address: Ruediger. Jehn@esa.int (R. Jehn).
} 


\section{INTRODUCTION}

BepiColombo will be the first European mission to Mercury and the first mission ever to use solar electric propulsion (SEP) to reach an inner planet. The interplanetary trajectory, consisting of a combination of low-thrust arcs and seven flybys (one at the Earth, two at Venus and four at Mercury), is a challenge in terms both of mission design and navigation. At the end of the transfer, a gravitational capture at the weak stability boundary of Mercury is performed exploiting the Sun gravity. Several aspects have to be considered when analysing the feasibility of each proposed interplanetary trajectory from the navigation point of view:

- Solar electric propulsion increases the flexibility and number of possible transfers, also allowing partial redesign of the trajectory at very low propellant costs. On the other hand it is also a source of noise for the spacecraft dynamics than can negatively affect the orbit determination from Earth. Because of this, 30-day coast arcs (without lowthrust propulsion) are introduced prior to all flybys to minimise the risks during critical phases of the mission.

- The low altitude of the second Venus gravity assist (minimum altitudes of $300 \mathrm{~km}$ ) and the Mercury gravity assists (at $200 \mathrm{~km}$ ), and the gravity capture at the arrival [7] lead to critical phases requiring very precise navigation.

- The event time scales of inner solar system missions are considerably smaller than for missions to the outer planets, which means that planetary encounters will take place closer to each other. This reduces the length of routine phases and the available time for precise determination and correction of errors between events.

- The duration of solar conjunctions is smaller for similar reasons, but at the same time they happen more often (one superior conjunction every approximately 120 days in the orbit of Mercury). During a few days radiometric measurements are severely degraded due to the passage of the radio signals through the solar plasma [11], and no safe up-link of telecommands can be guaranteed.

- Ground station coverage will be limited for cost reasons.

- Solar radiation pressure (SRP) forces are up to eleven times larger in the vicinity of Mercury when compared to Earth. Depending on the spacecraft design, the torque introduced by the solar radiation pressure can quickly saturate the momentum wheels, requiring more frequent momentum wheel-off-loading burns. In the case of an unbalanced reaction control system (RCS), these burns may also introduce perturbations in the trajectory.

- Navigating through the weak stability boundary introduces further challenges: small errors in velocity may translate into large errors in the final position when propagating in the vicinity of the Lagrangian points.

\section{REFERENCE TRAJECTORY}

The current baseline trajectory foresees a launch in July/August 2014. Tables 1 and 2 give the details of the interplanetary trajectory [6]. The launch window opens on 19 July 
Table 1

Main characteristics for the 6.3-year trajectory with up to $290 \mathrm{mN}$ thrust.

\begin{tabular}{|llcc|}
\hline Departure & Launch Date & 19 July 2014 & (MJD2000 = 5313) \\
& Escape Velocity & $3.36 \mathrm{~km} / \mathrm{s}$ & \\
& Escape Declination & $-3.8^{\circ}$ & \\
& Initial mass & $4040 \mathrm{~kg}$ & \\
\hline Arrival & Date & $13 \mathrm{Nov} 2020$ & (MJD2000 = 7622.66) \\
& Mercury true anomaly & $67.8^{\circ}$ & \\
& Velocity at periherm & $3.881 \mathrm{~km} / \mathrm{s}$ & $(2880 \times 180440 \mathrm{~km})$ \\
& $\Omega_{\text {arr }}$ & $67.7^{\circ}$ & (approach over \\
& $\omega_{\text {arr }}$ & $-2^{\circ}$ & South pole) \\
\hline Delta-V & SEP & $3.844 \mathrm{~km} / \mathrm{s}$ & \\
\hline SEPM & Specific Impulse & $4200 \mathrm{~s}$ & \\
& Maximum thrust level & $290 \mathrm{mN}$ & \\
& Total Impulse & $14.8 \mathrm{MNs}$ & \\
\hline
\end{tabular}

2014. Ariane 5 launches the spacecraft with an escape velocity of $3.36 \mathrm{~km} / \mathrm{s}$ and an escape declination of $-3.8^{\circ}$ which is optimum for the launcher performance. Since the spacecraft is expelled mainly radially inwards with respect to the Earth velocity vector, it will first travel inside $1 \mathrm{AU}$ (being visible at the ground stations at daytime). A long accelerating thrust arc of about 4 months moves the spacecraft outside 1 AU where a braking arc brings the spacecraft back to the Earth where a flyby finally provides the required rotation of the velocity to reach Venus on a ballistic arc 174 days later. Since the maximum deflection angle is only about $45^{\circ}$ for a Venus flyby at $9 \mathrm{~km} / \mathrm{s}$, two Venus flybys (separated by 1 Venus year, i.e. 225 days) are necessary to rotate the velocity vector into the optimum direction (mainly backwards with respect to the velocity of Venus).

During 2.5 heliocentric revolutions two small braking arcs after aphelion prepare for the first Mercury flyby. Thereafter the spacecraft passes through a 3:2 resonance and 5:4 resonance with Mercury. The last two Mercury flybys are within 40 days (the first close to Mercury's perihelion and the second one $180^{\circ}$ later) as proposed by Langevin [9]. They propel the spacecraft into the required orbital plane and reduce the relative velocity down to $1.9 \mathrm{~km} / \mathrm{s}$. Six final thrust arcs further reduce the relative velocity such that the spacecraft will be weakly captured by Mercury on 13 November 2020 even if no orbit insertion manoeuvre takes place. The total interplanetary cruise $\Delta \mathrm{V}$ is $3.844 \mathrm{~km} / \mathrm{s}$, all delivered by SEP. Fig. 1 shows the thrust profile as function of time and Fig. 2 shows the trajectory projected onto the ecliptic plane. 


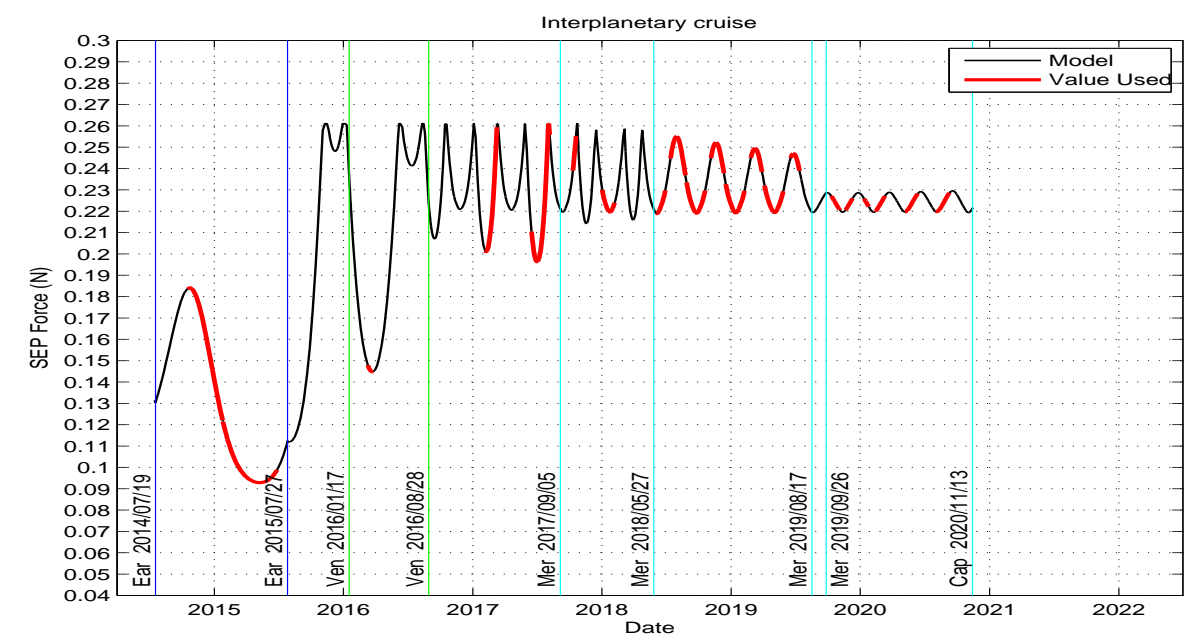

Fig. 1. Thrust profile during the interplanetary transfer.

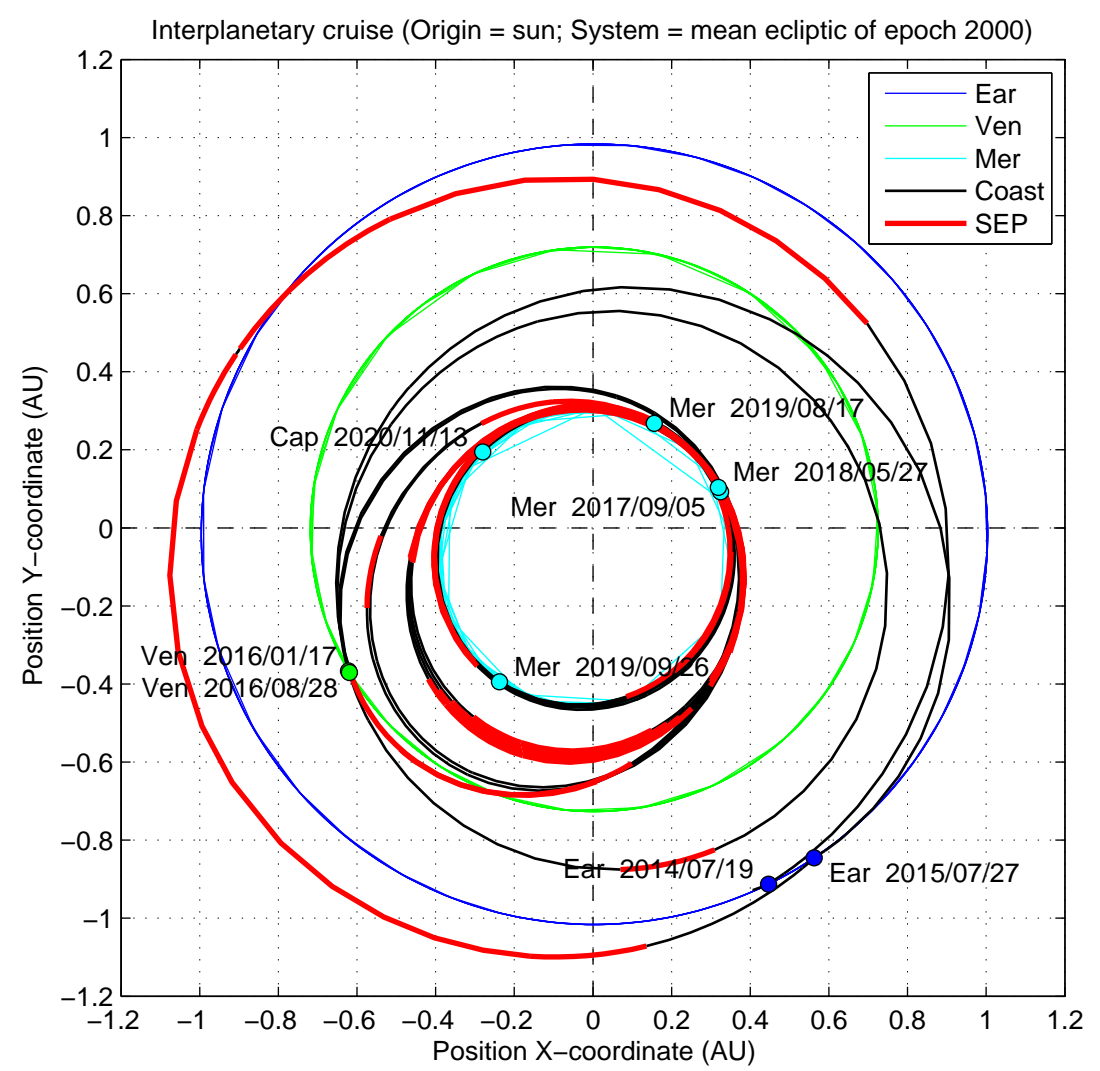

Fig. 2. Trajectory with a launch on 19 July 2014 and gravitational capture on 13 Nov 2020.

Of particular interest for the navigation analysis is Fig. [3], showing the trajectory in a Sun-Earth fixed coordinate system, and Table 3. The latter provides the details of the periods of solar conjunctions along the interplanetary trajectory. It can be seen that a 7day conjunction starts 5 days after Mercury- 2 flyby and right in the middle of the Mercury 3 and 4 flybys there is a 10-day conjunction. The arrival takes place 27.5 days before the next conjunction (17 days within 5 deg of the Sun) which has an impact on the sequence of the Mercury orbit insertion manoeuvres. 
Table 2

Flyby characteristics of the interplanetary trajectory. $\lambda_{S u n}$ is the solar longitude, $\mathrm{v}_{\infty}$ is the velocity at infinity, $\mathrm{h}_{p}$ is the pericentre altitude and $\gamma_{\text {defl }}$ is the deflection angle of the flyby.

\begin{tabular}{lccccc}
\hline & Date & $\lambda_{\text {Sun }}$ & $\mathrm{v}_{\infty}$ & $\mathrm{h}_{p}$ & $\gamma_{\text {defl }}$ \\
& & {$[\mathrm{deg}]$} & {$[\mathrm{km} / \mathrm{s}]$} & {$[\mathrm{km}]$} & {$[\mathrm{deg}]$} \\
\hline $\mathrm{E}$ & $2015-07-27$ & -56.4 & 4.26 & 3510 & 87.3 \\
V1 & $2016-01-17$ & -149.5 & 9.16 & 1351 & 40.2 \\
V2 & $2016-08-28$ & -149.1 & 9.22 & 300 & 44.1 \\
M1 & $2017-09-05$ & 15.8 & 5.63 & 200 & 24.0 \\
M2 & $2018-05-27$ & 18.0 & 5.34 & 200 & 26.2 \\
M3 & $2019-08-17$ & 59.8 & 2.82 & 200 & 61.7 \\
M4 & $2019-09-26$ & -121.1 & 1.89 & 200 & 88.8 \\
\hline
\end{tabular}

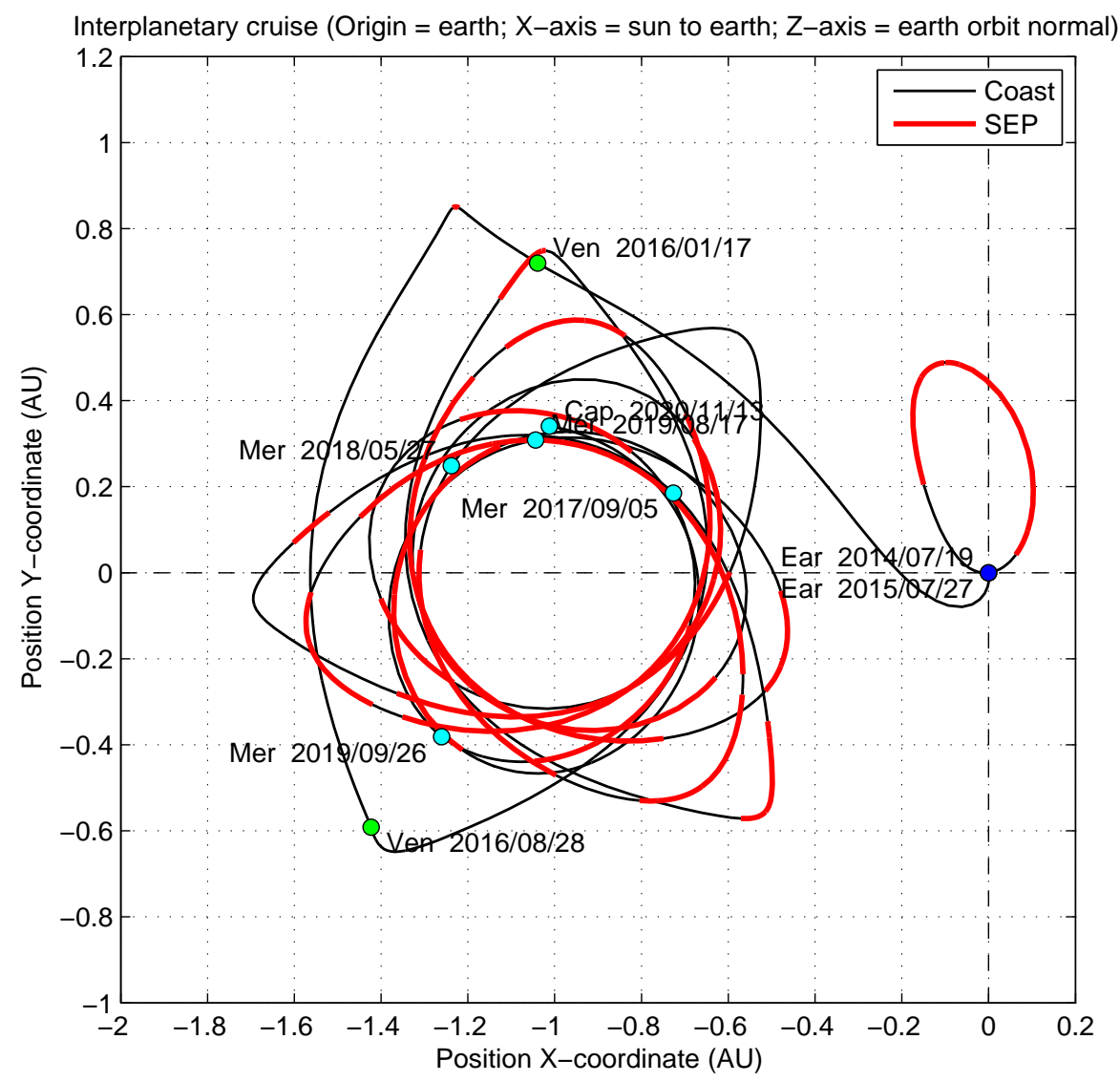

Fig. 3. Interplanetary trajectory of BepiColombo plotted in a Sun-Earth fixed coordinate system. The thrust arcs are shown as thick red lines. 
Table 3

Periods of superior solar conjunction during the interplanetary trajectory (a critical Sun-EarthSC angle of $5.0^{\circ}$ and $2.8^{\circ}$ is assumed).

\begin{tabular}{ccrccr}
\hline \multicolumn{3}{c}{ Critical angle: 5.0} & \multicolumn{4}{c}{ Critical angle: 2.8 degrees } \\
Begin & Duration & \multicolumn{2}{c}{ Begin } & Duration \\
Calendar & MJD2000 & (days) & Calendar & MJD2000 & (days) \\
\hline $2016 / 7 / 7$ & 6032.2616 & 12.710 & & & \\
$2017 / 4 / 11$ & 6310.9743 & 8.097 & $2017 / 4 / 13$ & 6312.8862 & 4.394 \\
$2017 / 10 / 12$ & 6494.2871 & 55.642 & $2017 / 10 / 19$ & 6501.6722 & 32.638 \\
$2018 / 6 / 1$ & 6726.4630 & 7.289 & $2018 / 6 / 3$ & 6728.1052 & 3.998 \\
$2018 / 10 / 20$ & 6867.0193 & 26.578 & $2018 / 10 / 24$ & 6871.6473 & 14.608 \\
$2019 / 4 / 23$ & 7052.8587 & 8.190 & $2019 / 4 / 25$ & 7054.7594 & 4.541 \\
$2019 / 8 / 30$ & 7181.1470 & 10.415 & $2019 / 9 / 1$ & 7183.4377 & 5.499 \\
$2019 / 12 / 31$ & 7304.7321 & 14.521 & $2020 / 1 / 5$ & 7309.0829 & 6.297 \\
$2020 / 4 / 27$ & 7422.3680 & 8.461 & $2020 / 4 / 29$ & 7424.2937 & 4.698 \\
$2020 / 8 / 11$ & 7528.7313 & 9.371 & $2020 / 8 / 14$ & 7531.1762 & 4.262 \\
\hline
\end{tabular}

\section{NAVIGATION ASSUMPTIONS AND MODELS}

A batch-sequential Square Root Information Filter (SRIF) based on Bierman's formulation [2], as implemented in the tools INTNAV [1] and LOTNAV [3], was used to process the measurements with a batch size of 0.5 days.

Two-way X-band (8.4-Ghz) Doppler and range data are acquired from a single station in Cebreros (Spain) and $\triangle \mathrm{DOR}$ measurements from Cebreros, New Norcia (Australia) and a third deep space antenna in Argentina are scheduled before planetary flybys when required. During the interplanetary phase measurements are taken only once per week. Intense measurement campaigns begin 30 days prior to each flyby (lasting until a postflyby cleanup manoeuvre is performed), and also 30 days before the beginning of a lowthrust arc. During these phases daily measurements are taken.

Several error sources are accounted for in the filter, either as exponentially correlated random variables (ECRV, for the solar radiation pressure, non-gravitational accelerations, and SEP thrust modulus and direction), Gaussian errors (for the implementation of trajectory correction manoeuvres -TCMs-, and measurement noises) or as considered biases (in the station location and range measurements). Tables 4 and 5 summarise the assumptions and error models adopted. Note that all the values are conservative, in some cases over one order of magnitude higher than current accuracies or known error sources. A more detailed description of the assumptions can be found in [4]. 
Table 4

Measurement model assumptions. $\triangle \mathrm{DOR}$ measurements are only scheduled in critical phases and before planetary flybys when required.

\begin{tabular}{lccccc}
\hline & Ground Stations & Freq. & $1 \sigma$ error & Bias & Min. Elev \\
\hline Range & Cebreros & $60 \mathrm{~min}$ & $10 \mathrm{~m}$ & $2 \mathrm{~m}$ & $10^{\circ}$ \\
Doppler & Cebreros & $10 \mathrm{~min}$ & $0.3 \mathrm{~mm} / \mathrm{s}$ & - & $10^{\circ}$ \\
$\Delta$ DOR & Cebreros-New Norcia & 4 days & $0.1 \mathrm{~m}$ & - & $15^{\circ}$ \\
\hline
\end{tabular}

Table 5

Noises and error assumptions.

\begin{tabular}{cccc}
\hline & Type & $1 \sigma$ error & Correlation time \\
\hline SRP & ECRV & $10 \%$ & $10 \mathrm{~d}$ \\
NGA & ECRV & $10^{-11} \mathrm{~km} / \mathrm{s}^{2}$ & $1 \mathrm{~d}$ \\
SEP mod. & ECRV & $1 \%$ & $1 \mathrm{~d}$ \\
SEP ang. & ECRV & $0.5^{\circ}$ & $1 \mathrm{~d}$ \\
TCM mod. & Gauss & $1 \%$ & - \\
TCM dir. & Gauss & $0.5^{\circ}$ & - \\
Stat. Loc. & Bias & $1 \mathrm{~m}$ in $\mathrm{x}$ and $\mathrm{y}, 2 \mathrm{~m} \mathrm{in} \mathrm{z}$ \\
\hline
\end{tabular}

Two different types of guidance strategies were implemented for the navigation analysis:

1. During coast arcs, trajectory correction manoeuvres are computed using a fixed time linear guidance algorithm [1], normally aiming at the pericentre. These trim manoeuvres reduce the dispersion to the current level of knowledge (both dispersion and knowledge are propagated by means of a modelled-world transition matrix to the target point). As it is shown in Table 5, Gaussian errors are assumed for the execution of these TCMs both in modulus and in pointing. Due to these errors, due to further improvement of the knowledge, and due to the fact that the propagation of the covariance matrices in the real world takes into account all other implemented noises, more than one TCM is normally beneficial to clean up errors of the previous ones and to reduce the increasing dispersion. For pure ballistic phases between flybys, a TCM is scheduled shortly after the first flyby, in order to compensate the errors in the flyby execution. Two more manoeuvres are scheduled during the last 30 days of approach. Although covariance analyses could be performed for coast arcs (which indeed were performed for test purposes), Monte-Carlo simulations were performed to harmonize the approach with the navigation analysis for thrust arcs.

2. During thrust arcs, the control parameters of the thrust law are modified with a linearquadratic control (LQC) algorithm. The thrust control law (modulus and angles) is expressed as a combination of Chebyshev polynomials, and the linear-quadratic controller 
modifies the coefficients of the polynomials with the aim of reducing the dispersion at the end of that thrust arc to the level of knowledge. Due to the non-linearity and complexity of the problem, the guidance analysis of the phases with low-thrust arcs are performed with a Monte-Carlo simulation tool [3] and its results are later statistically processed to obtain the guidance costs, as well as the final dispersion and knowledge ellipsoids. When coming from a thrust arc, besides the low-thrust navigation and guidance law, two or three trim manoeuvres are scheduled previous to the planetary encounter. No post-correction TCM is scheduled after a flyby if a thrust arc is following immediately.

\section{NAVIGATION ANALYSIS}

The results of a detailed navigation analysis for the first phases of the 2013 BepiColombo trajectory were presented in [5]. The sum of the 3-sigma trajectory correction manoeuvres was $88 \mathrm{~m} / \mathrm{s}$. However, except for the correction manoeuvre V1-20 (11.6 m/s twenty days before the first Venus flyby) and M1+5.5 (25.3 m/s five and a half days after the first Mercury flyby), all correction manoeuvres could be performed with low thrust. Since there are solar aspect angle constraints, the manoeuvres may have to be decomposed in two manoeuvres ("dog-leg" manoeuvre) which can increase the required delta-V by a factor of up to 4.8 in case of a solar aspect angle constraint of $12 \mathrm{deg}$. On top of the $154 \mathrm{~m} / \mathrm{s}$ navigation delta- $\mathrm{V}$ required during the low-thrust arcs, another $170 \mathrm{~m} / \mathrm{s}$ was required for these correction burns [8]. A detailed list of the interplanetary navigation delta-Vs for the April 2014 trajectory is given in the final report of the UMAST project [10].

In this paper the navigation analysis of the final part of the 2013 trajectory is presented in detail. Fig. shows the final approach towards Mercury. The spacecraft passes close to $L_{2}$ before "sliding" through $L_{1}$ into a gravity capture at Mercury. The last 60 days are without thrust and no nominal manoeuvre. A trajectory correction manoeuvre is scheduled 65 days before the arrival to Mercury at the end of the last thrust arc. It is intended to reduce the high initial dispersion. Four additional TCMs are scheduled during the gravitational capture. During this phase an initial mass of $1300 \mathrm{~kg}$ and an effective surface of $10 \mathrm{~m}^{2}$ is considered after releasing the SEP module. The best dates and target points for these manoeuvres were determined by parametric analysis. A solar conjunction takes place shortly before Mercury Orbit Insertion (the spacecraft is within $2.8 \mathrm{deg}$ from the centre of the Sun from 16 to 12 days before MOI). It is assumed that no measurements and no manoeuvres are performed during these 4 days. Operational constraints impose seven days of precise orbit determination after the solar conjunction and before any manoeuvre takes place. Three days before the solar conjunction are also allocated for possible additional corrections. These constraints fix the dates of the last two manoeuvres at MOI-20 and MOI-5.

The first two manoeuvres are needed to reduce the high initial dispersion that comes from the interplanetary trajectory and are almost independent of the error assumptions in the dynamics. The TCM at MOI-30 is basically a clean-up of the errors in the previous TCM 


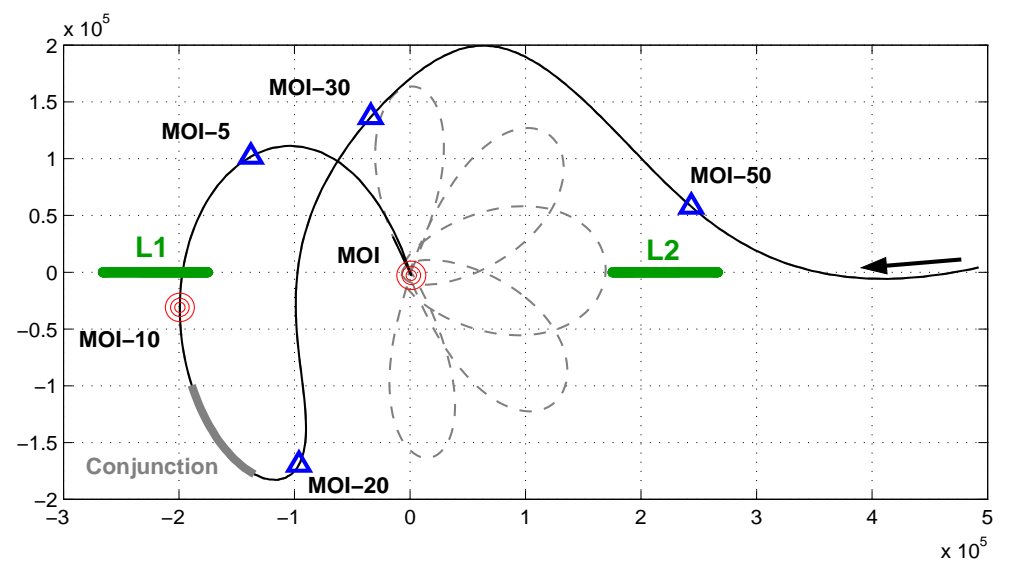

Fig. 4. Gravitational capture approach in a co-rotating frame. The negative $\mathrm{X}$-axis points towards the Sun. L1 and L2 points move due to the eccentricity of Mercury's orbit. Trajectory correction manoeuvres are indicated with triangles, and the targeting points with circles. The part of the trajectory during solar conjunction is shaded.

Table 6

Navigation delta-Vs (in $\mathrm{m} / \mathrm{s}$ ) during the final Mercury approach. 3-sigma values $(99.7 \%$ ) are given.

\begin{tabular}{cccc}
\hline MJD & TCM & Target & $\Delta \mathrm{V}$ \\
\hline $\mathbf{7 0 3 0}$ & MOI-65 & MOI-10 & 9.64 \\
$\mathbf{7 0 4 5}$ & MOI-50 & MOI-10 & 4.79 \\
$\mathbf{7 0 6 5}$ & MOI-30 & MOI-10 & 0.72 \\
$\mathbf{7 0 7 5}$ & MOI-20 & MOI & 0.30 \\
$\mathbf{7 0 9 0}$ & MOI-5 & MOI & 5.18 \\
\hline
\end{tabular}

execution. The size of the manoeuvre at MOI-5 is directly influenced by the errors in the dynamics in the weak stability boundary. SRP errors are found to be more relevant than NGA (probably because of a higher correlation time). A better knowledge or estimation of the SRP acceleration can reduce the TCM-MOI-5 size by one third. The inclusion of a small manoeuvre at MOI-20 is also very useful to reduce the error build-up caused by SRP and NGA. Higher execution errors (up to $50 \%$ in size) were considered for such a small manoeuvre and the improvement was still relevant. It might be possible to use periodical wheel desaturation burns to perform these small corrections. An additional manoeuvre at MOI-2 can be considered to correct errors from TCM-MOI-5, although in principle it is not required. It was also found that targeting to Mercury periherm more than 30 days before MOI is not the best strategy. Targeting at that time to a point closer to $L_{1}$ proved to be more effective. Table 6 summarises the results for the trajectory correction manoeuvres. The time and position of the correction manoeuvres and targeting points are also indicated in Fig. [4]. 
Results from the navigation analysis indicate that an allocation of $20 \mathrm{~m} / \mathrm{s}$ for correction manoeuvres during the gravitational capture is required. The last three manoeuvres have to be performed with the chemical engine, as the SEP module is jettisoned before the capture.

Fig. 5 shows the evolution of the dispersion and knowledge in position between the last Mercury flyby and the final orbit insertion. During the low-thrust arcs (shaded regions) the position knowledge degrades. The along-track and radial uncertainty decreases after each measurement once a week but the cross-track error keeps increasing. As soon as the thrust is interrupted, the knowledge improves by more than one order of magnitude. There are daily measurements during the final coast arcs and $\triangle \mathrm{DOR}$ measurements from the Cebreros-New Norcia baseline every four days starting after the last thrust arc. As a consequence, the position uncertainty decreases below $10 \mathrm{~km}$ (1-sigma).
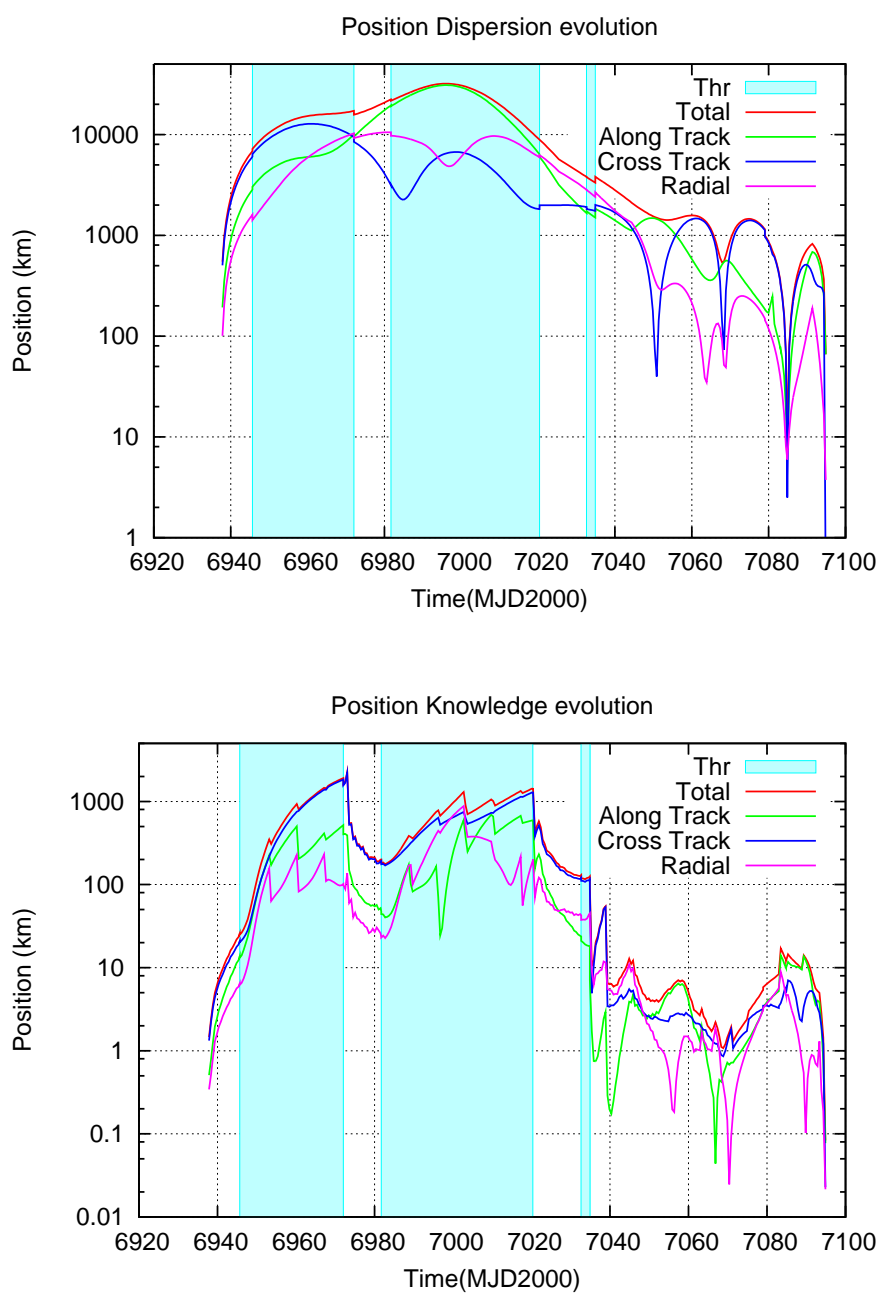

Fig. 5. $1 \sigma$ Along-track, cross track and radial position dispersion (above) and knowledge (below) between 2nd Mercury flyby and MOI. Low-thrust arcs are shaded. 
Fig. 6 shows the evolution of the velocity knowledge and dispersion. During thrust arcs the velocity can only be determined with a precision of $1 \mathrm{~m} / \mathrm{s}$. However with an increased tracking frequency and $\triangle \mathrm{DOR}$ measurements the precision drops to a level of $1 \mathrm{~cm} / \mathrm{s}$ after the last thrust arc before it starts increasing again prior to orbit insertion. With the presented manoeuvre strategy, an accuracy of $11 \mathrm{~km}$ (3-sigma) in cross-track and radial position is achieved at orbit insertion.
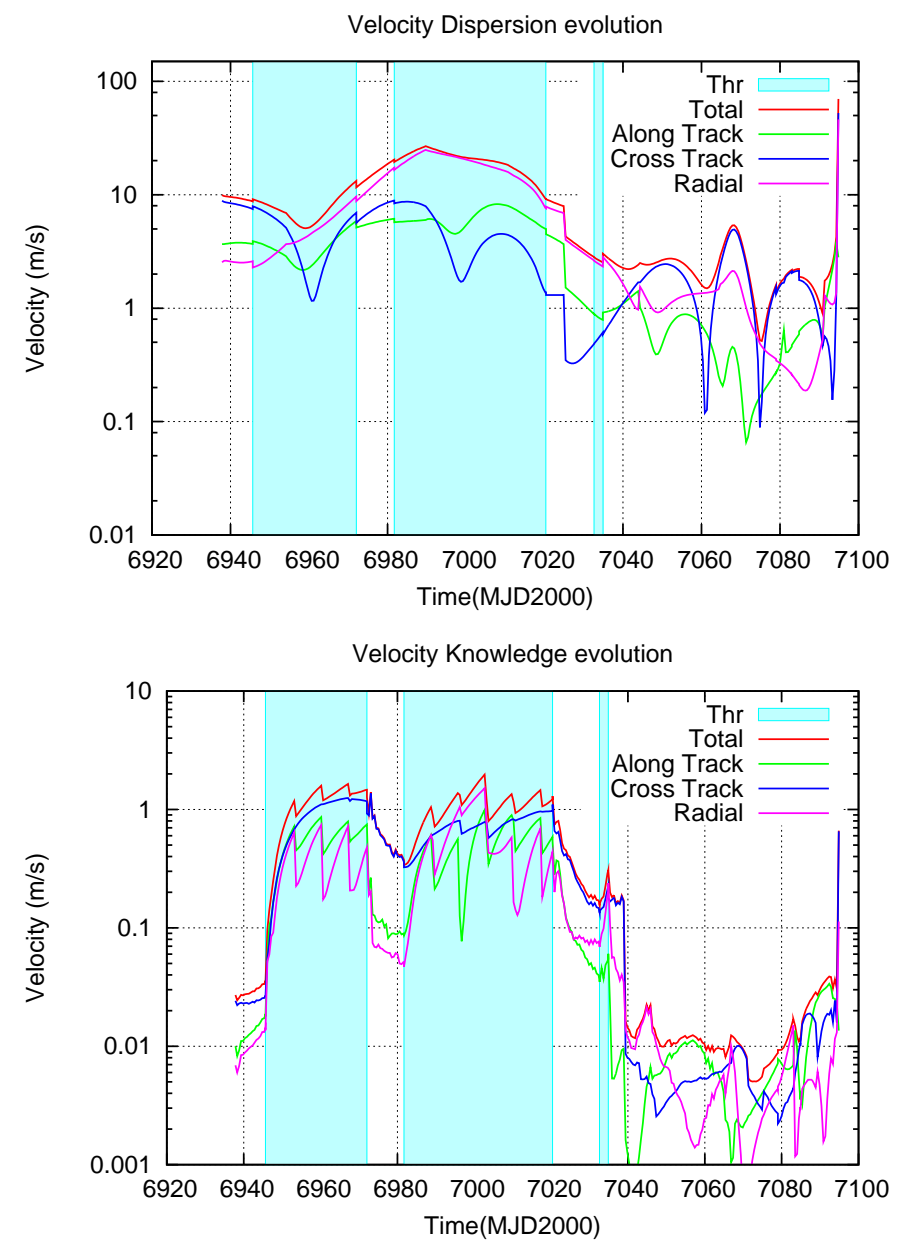

Fig. 6. $1 \sigma$ Along-track, cross track and radial velocity dispersion (above) and knowledge (below) between 2nd Mercury flyby and MOI. Low-thrust arcs are shaded.

\section{CONCLUSIONS}

The combination of flybys, solar conjunctions and different sources of noise must be taken into account in the trajectory design and the navigation analysis of the BepiColombo mission to Mercury. Performing low-thrust navigation by modifying the thrust law once per week has proved to be an efficient method of reducing the dispersion even with very conservative assumptions. 
Nevertheless, the orbit knowledge is strongly degraded during low-thrust arcs and therefore 30-days coast arcs are introduced prior to any flyby to allow for a better orbit determination and orbit correction. Although the final approach through the $L_{2}$ and $L_{1}$ point seems risky from a navigation point-of-view, it turned out that a very robust navigation is possible with daily tracking from one ground station. Adding $\triangle$ DOR measurements will make the approach navigation even safer and will reduce the propellant requirements. Currently 5 trajectory correction manoeuvres with a maximum of $20 \mathrm{~m} / \mathrm{s}$ (3-sigma) are scheduled.

The navigation analysis has demonstrated that BepiColombo is on a safe track, in spite of its complex trajectory.

\section{References}

[1] Belló M. and Baeza M., Software Tool for Interplanetary Navigation Final Report G.M.V., ESA Contract No. 9715/91/D/IM, 1992.

[2] Bierman G.J., Factorization Methods for Discrete Sequential Estimation, Academic Press, 1977.

[3] Cano J. L. and Belló M., Final Report on the Development of the LOTNAV Tool, Deimos-Space, ESA Contract No. 16650/02/D/KH, 2004.

[4] García Yárnoz D., Jehn R. and Croon M., Interplanetary navigation along the lowthrust trajectory of BepiColombo, Acta Astronautica, Vol. 59, pp. 284-293, 2005.

[5] García Yárnoz D., Jehn R. and Sánchez N., BepiColombo: Navigation challenges on the way to Mercury, 25th Int. Space Technology and Science Conference, Kanazawa, Japan, 4-11 June 2006.

[6] García Yárnoz D., Jehn R., Schoenmaekers J. and de Pascale P., BepiColombo Mercury Cornerstone Consolidated Report on Mission Analysis, MAS Working Paper 525, BC-ESC-RP-05500, Issue 3.0, March 2009.

[7] Jehn R., Campagnola S., García Yárnoz D. and Kemble S., Low-thrust approach and gravitational capture at Mercury, 18th Int. Symposium on Space Flight Dynamics, Munich, Germany, 11-15 Oct. 2004.

[8] Jehn R., Companys V., Corral. C., García Yárnoz D. and Sánchez N., Navigating BepiColombo during the weak-stability capture at Mercury, Adv. Spa. Res. 42, pp. 1364-1369, 2008.

[9] Langevin Y., Chemical and solar electric propulsion options for a Cornerstone Mission to Mercury, Acta Astronautica, Vol. 47, Nos. 2-9, pp. 443-452, 2000.

[10] Sánchez N., Belló M., Cano J.L. and Martin L., Upgrade of Mission Analysis Software Tools to Study Low-Thrust Planetary Exploration Missions, ESA/ESOC Final Report of Contract 19511/05/D/HK, Deimos, Madrid, March 2009.

[11] Timm R., Solar Plasma Influence on Ranging Accuracy, BC-MA-TN-1001-TOS-OSF, Issue 1.2, ESA/ESOC, August 2002. 